\title{
Inverse Bilateral Filter for Saliency
}

\author{
Dao Nam Anh \\ Department of Information Technology \\ Electric Power University \\ 235 Hoang Quoc Viet road \\ Hanoi, Vietnam
}

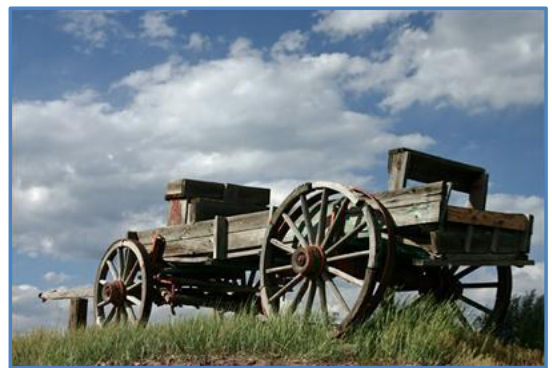

a. Input 197_197544.jpg

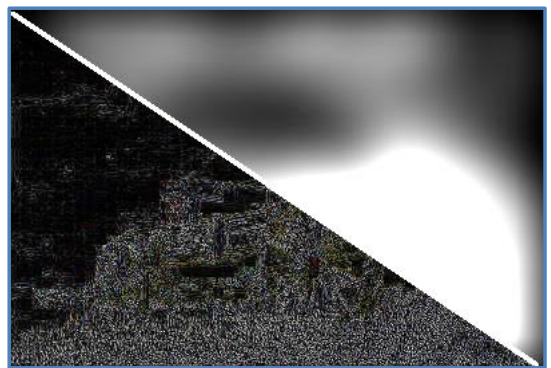

b. Contrast by the inverse bilateral filter (bottom left) and saliency map (top

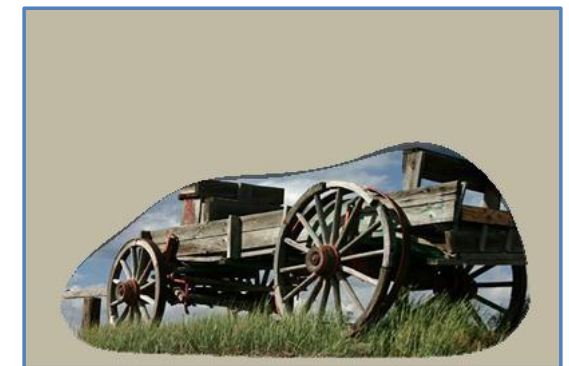

c. Extracted salient region right)

Figure 1: Example of extracting salient region by the inverse bilateral fitter

\begin{abstract}
The analysis and automatic detection of visual salient image regions has been the subject of considerable research useful in object segmentation, adaptive compression and re-targeting. However, the nature of the essential mechanisms intervening human visual saliency remains elusive. To assess the validity of salient regions some kind of prior model is consistently required. This paper proposes a new model using inverse bilateral fitter that allows the system to output saliency maps with salient objects in their context. The filter is described firstly for automatically learning local contrast distribution to accurately predict salient image regions. Along with the contrast distribution checking, local opposition is analyzed by the second application of the inverse bilateral filter to establish fuzzy boundary of salient regions in form of trimap. This approach is shown to increase the reliability of identifying visual salient objects. Output from the research has potential applications in the areas of object detection and recognition.
\end{abstract}

\section{General Terms}

Pattern Recognition, Algorithms.

\section{Keywords}

Inverse bilateral filter, saliency, contrast, trimap.

\section{INTRODUCTION}

Visual target detection is a procedure of decomposing image into object, person or regions of having relative distinction to their neighbors, offering a powerful representation for attention capture. The chosen salient regions should allow the resulting region to be homogeneous within and heterogeneous between regions. This technique also applies to image segmentation for image editing [1], image cropping for browsing [2], object recognition [3], and content-aware image resizing [4]. In particular the saliency detection is important for adaptive content delivery [5], adaptive region-of-interestbased image compression [6][7], video summarization [8] and media retargeting [9][10].

The visual target detection models can be described in three categories: a) those targeting to identify and separate the most salient object in a scene, b) active segmentation approaches, and c) models that address fixation prediction [11].

The motivation for this paper is a system for automatically archiving and segment the most visual object corresponding to the first category above with a strong similar nature within region and robust diversity between regions. The major obstacle for the system is the vast range of content and volatility of image properties. The scenes are captured under many different circumstances inducing considerable dissimilarity in texture and particularly color.

Hence this paper concentrates purely on contrast and opposition analysis by two steps to getting visual saliency high level. Drawing on the principle that a salient region is defined as homogeneous contrast level within, i.e. inside the region, a novel approach to discover contrast level is introduced by the inverse bilateral filter $(\mathrm{BF})$. As original $\mathrm{BF}$ [12][13][14] is based on similarity of intensive level of a pixel with its neighbor, our approach offers inverse BF that is based on distinction instead of similarity.

Consequently, contrast level can be detected by inverse BF and analyzed for saliency in the first step. The second step applies the same inverse $\mathrm{BF}$ on the contrast level to get opposition degrees, which is to define heterogeneous measure. Then each image pixel is grouped into salient regions with fuzzy borders defined by both contrast and opposition levels. An example for this process is outlined in Fig 1: an image (Fig.1a) from MSRA Salient Object Database [15] get its inverse BF's result in Fig.1b (bottom) and saliency map in Fig.1b (top). Visual target is extracted in Fig.1c.

Our contributions are three-fold. First, a new principle of inverse bilateral filter is introduced. Second, based on the principle, a new saliency detection method is presented and accuracy and reliability are determined. An algorithm for computing saliency map representations is shown. Third, a comparative study on the results of the inverse BF method and alternative saliency methods on the same data set is demonstrated. 


\section{OUTLINE OF PAPER}

A brief outline of the paper is as follows: Section 3 describes prior work in saliency detection. Section 4 presents the inverse bilateral filter and discusses its use in saliency detection. An overview of the IBIS algorithm is described in Section 4.4. The experiments of the algorithm and discussion are covered in Section 6, and Section 7. Finally, future works are shown in Section 8, followed by conclusion in Section 9.

\section{PRIOR WORK}

In this section, saliency detection techniques are firstly reviewed and applications of bilateral filter is highlighted. Then issues associated with saliency systems, in particular contrast representations is discussed.

\subsection{Saliency detection techniques}

A common aspect of how attention is deployed onto a given scene is feature-based saliency. Treisman et al [15] suggests that visual search paradigm allows to define a target either by its separate features or by their conjunction. Thus, saliency is attributed to different features. An approach in [17] combines feature maps, from different visual modalities such as color and orientation, into a unique saliency map. Non-spatial, feature-based intentional modulation of visual motion processing was demonstrated in [18]. Thus, attention increases the gain of direction-selective neurons in visual cortical area.

Visual saliency is studied like space-based, where saliency is deployed at different locations. The spatial deployment of the limited-capacity process get its attention, and intentional control of limited resources is guided by the output of other earlier parallel processes in [19]. A solution to the problems of saliency detection by routing information through a visual processing with hierarchy and task-specific intentional bias is shown in [20]. Region saliency is proposed to be based on space and motion, and scale space analysis of the log amplitude spectrum of natural images and videos is noted in [21].

Several models have been proposed in object-based aspect where saliency is deployed on different objects or groups. An approach in [22] states that the units of attention are often various kinds of visual objects. Interactive dynamics of object and spatial contextual cueing and attention in the cortical are presented in [23][24]. A model of proto-objects that eventually guides a saliency mechanism is defined in [25] and applied on a humanoid robot. Results of [26] shows that the object-based interpretation of saliency can be a predictor of fixation locations.

\subsection{Applications of bilateral filter}

Bilateral filter introduced in [12] is an effective smoothing filter that preserves edges. An approach is proposed for video de-blocking which performs perceptually adaptive bilateral filtering by considering color, intensity, and motion [27]. The approach uses a saliency map to control the strength of the filter for each individual point based on its perceptual importance.

Following content-aware saliency estimation in [28], a saliency rendering scheme is given by combining the saliency guided bilateral filtering and saliency guided contour detection techniques. Image fidelity is adjusted [29] before compression by using an extended bilateral filter, in which the local intensity and spatial scales are adjusted according to visual saliency.

The bilateral filter is iterated to simplify video content and achieve a cartoon look in [30]. The work suggests using saliency estimation to regulate bilateral filter's range weight. The spatial center and variances of the quantized colors are deal via an bilateral filtering and produces a probability of saliency based on a statistical object model [31]. An objectoriented saliency algorithm based on super-pixels rarity uses bilateral filter resulting an image with a cartoon-like effect [32].

The bilateral filter version considered in this work is particular special. It's the inverse version in comparison with the original version to manage saliency estimation. Next section presents it in details.

\section{INVERSE BILATERAL FILTER}

Let's start description by notation of color image, that can be represented by function $u(x)$ on the landmark coordinates $x$ :

$u(x): \Omega \rightarrow \mathfrak{R}^{3}$

Hence the saliency mapping function $s(x)$ can be modeled as:

$$
\begin{aligned}
& s(u): \Omega \rightarrow[0,1] \in \mathfrak{R}^{1} \\
& \left\{\begin{array}{l}
\Omega^{s}=\{x: s(u(x))=1\} \\
\Omega^{b}=\{x: s(u(x))=0\} \\
\Omega^{u}=\{x: 0<s(u(x))<1\}
\end{array}\right. \\
& \Omega=\Omega^{s} \cup \Omega^{b} \cup \Omega^{u}, \Omega^{s} \cap \Omega^{b}=\varnothing
\end{aligned}
$$

where $\Omega^{s}$ denotes salient region, $\Omega^{b}$-background and $\Omega^{u}$ - the blended region [33].

The saliency mapping function will be represented by inverse bilateral filer $\hat{B}(u)$ :

$$
s(u)=f(\hat{B}(u))
$$

\subsection{BILATERAL FILTER}

An important component of the $\mathrm{BF}$ is the Gaussian filter $G_{\sigma}$ which is a smoothing filter but at the cost of less distinct edges [34]:

$G_{\sigma}(x)=\frac{1}{\sigma \sqrt{2 \pi}} \exp \left(-\frac{x^{2}}{2 \sigma^{2}}\right)$

where $\sigma$ is filter scale.

Here is measure of intensive similarity for a pixel $x$ with other pixel $y$ :

$$
\operatorname{sim}(u)=\|u(\mathrm{x})-u(\mathrm{y})\|^{2}
$$

Thus the similarity of pixel $\mathrm{x}$ with its neighbor is represented by the Gaussian filter $G_{\sigma}$, applied on the intensive similarity:

$$
G_{\sigma}(\operatorname{sim}(u))=\frac{1}{\sigma \sqrt{2 \pi}} \exp \left(-\frac{\|u(x)-u(y)\|^{2}}{2 \sigma^{2}}\right)
$$

Contrast is opposite to the similarity as follows:

$\operatorname{contr}(u)=1-\operatorname{sim}(u)=1-\|u(\mathrm{x})-u(\mathrm{y})\|^{2}$

Contrast measure of pixel $x$ in considering its local neighbors $y$ now has its formula which is analogical to (7):

$$
G_{\sigma}(\operatorname{contr}(u))=\frac{1}{\sigma \sqrt{2 \pi}} \exp \left(-\frac{1-\|u(x)-u(y)\|^{2}}{2 \sigma^{2}}\right)
$$

Bilateral filter $B(u)$ [12][13][14] is based on similarity of intensive level $u$ of pixel $x$ with its neighbors $y$ :

$$
B(\mathrm{u})=\frac{1}{W_{\mathrm{x}}} \sum_{\mathrm{y} \in S} G_{\sigma_{s}}(\|\mathrm{x}-\mathrm{y}\|) G_{\sigma_{r}}(\operatorname{sim}(u)) u(\mathrm{y})
$$

Use (6) to replace $\operatorname{sim}(u)$ in (10): 
International Journal of Computer Applications (0975 - 8887)

Volume 118 - No. 10, May 2015

$B(\mathrm{u})=\frac{1}{W_{\mathrm{x}}} \sum_{\mathrm{y} \in S} G_{\sigma_{s}}(\|\mathrm{x}-\mathrm{y}\|) G_{\sigma_{r}}(\|u(\mathrm{x})-u(\mathrm{y})\|) u(\mathrm{y})$

where $\sigma_{s}$ and $\sigma_{r}$ are the spatial and intensity filter scales. $W_{x}$ is normalized weight at $x$ :

$W_{\mathrm{x}}(x)=\sum_{\mathrm{y} \in S} G_{\sigma_{s}}(\|\mathrm{x}-\mathrm{y}\|) G_{\sigma_{r}}(\|u(\mathrm{x})-u(\mathrm{y})\|)$

\subsection{CONTRAST FILTER}

Given contrast measure (8), new inverse bilateral filter is described analogically to (10):

$$
\hat{B}(\mathrm{u})=\frac{1}{\hat{W}_{\mathrm{x}}} \sum_{\mathrm{y} \in S} G_{\sigma_{s}}(\|\mathrm{x}-\mathrm{y}\|) G_{\sigma_{r}}(\operatorname{contr}(u)) u(\mathrm{y})
$$

Use (8) to replace contr( $u$ ) in (13):

$\hat{B}(\mathrm{u})=\frac{1}{\hat{W}_{\mathrm{x}}} \sum_{\mathrm{y} \in S} G_{\sigma_{s}}(\|\mathrm{x}-\mathrm{y}\|) G_{\sigma_{r}}(1-\|u(\mathrm{x})-u(\mathrm{y})\|) u(\mathrm{y})$

This is our final formal definition of inverse bilateral filter with its normalized weight $W_{x}^{\prime}$ :

$$
\hat{W}_{\mathrm{x}}(x)=\sum_{\mathrm{y} \in S} G_{\sigma_{s}}(\|\mathrm{x}-\mathrm{y}\|) G_{\sigma_{r}}(1-\|u(\mathrm{x})-u(\mathrm{y})\|)
$$

Denote local contrast estimation $c(x)$ for short:

$$
c(x)=\hat{B}(u(x))
$$

Thus, disparity $d(x)$ is simple difference of $u(x)$ and $c(x)$ :

$$
d(x)=\|u(x)-c(x)\|^{2}
$$

So, the disparity estimation $d(x)$ is result of applying the inverse bilateral filter on the input image $u(x)$.

\subsection{SALIENCY MAP DETECTION}

The procedure can be run again on $d(x)$ to get a higher level of contrast - the saliency estimation $v(x)$. Local opposition $o(x)$ is calculated similarly (but not identically) to (14) with input by $d(x)$ :

$$
o(x)=\hat{B}(d(x))
$$

Final saliency map $v(x)$ is the Euclidean distance of $d(x)$ and $o(x)$ :

$$
v(x)=\|d(x)-o(x)\|^{2}
$$

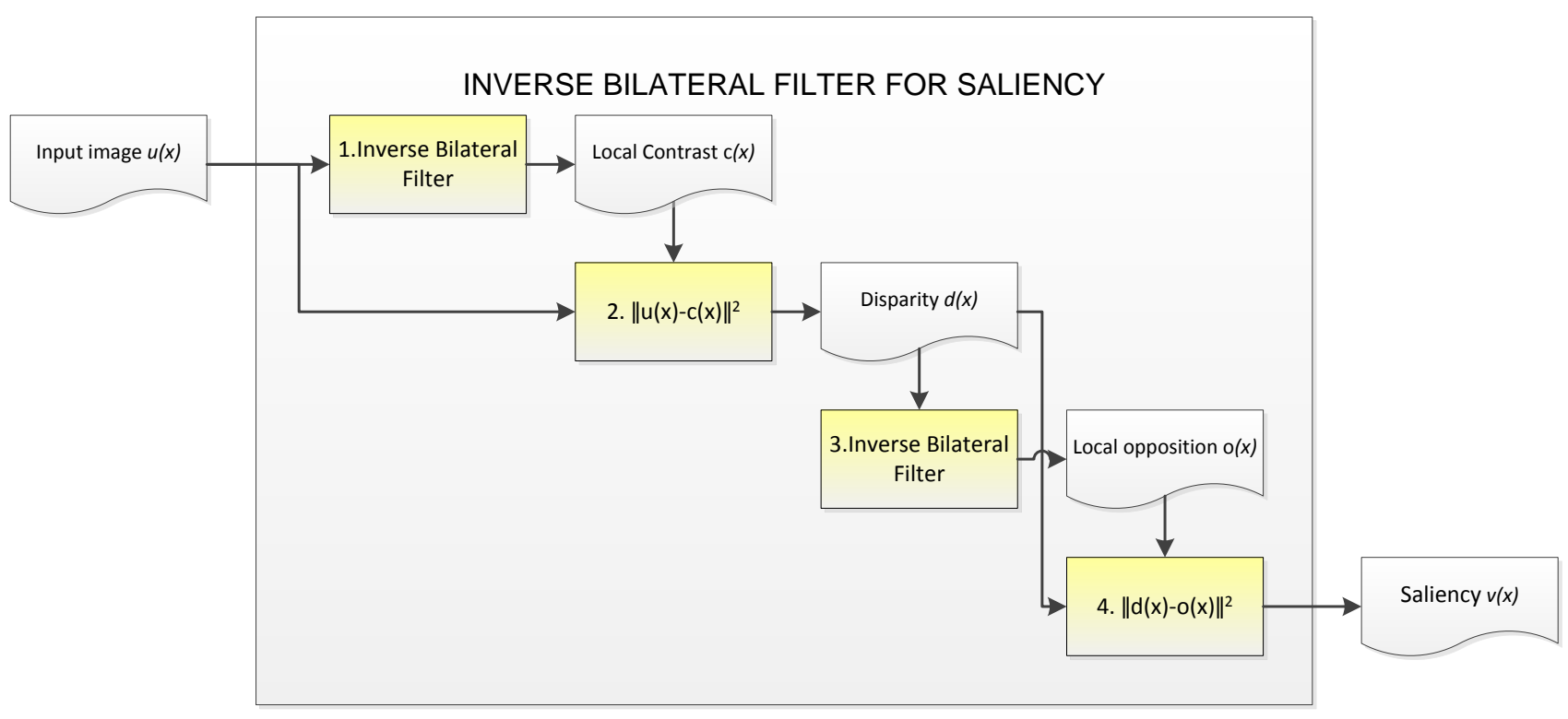

Figure 2: Algorithm of Inverse Bilateral Filter for Saliency Detection

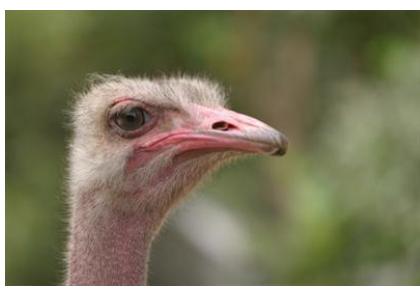

a) Input $u(x)$ by $192 \_192895 . j p g$

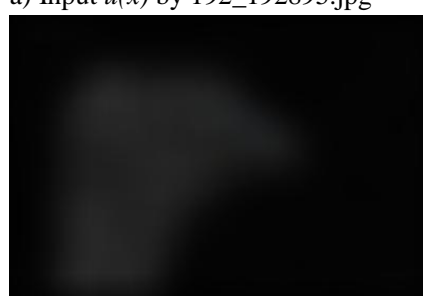

e) Saliency map $v(x)$

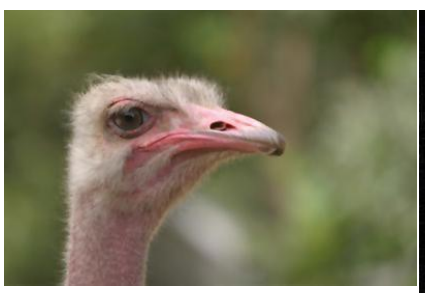

b) Inverse bilateral filter $c(x)$

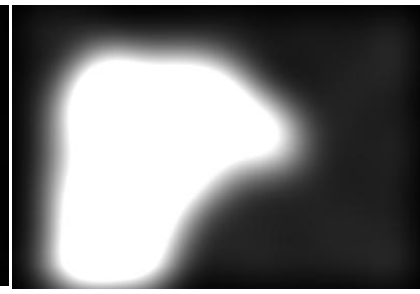

f) Normalized saliency map $v_{n}(x)$

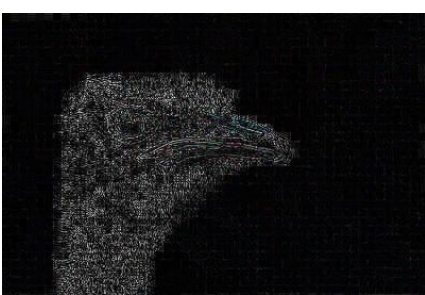

c) Disparity $d(x)$

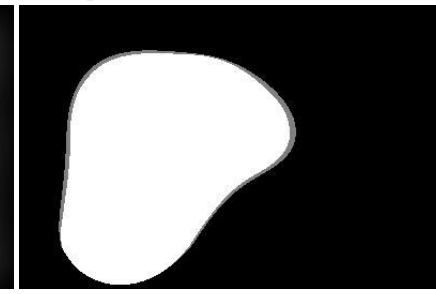

g) Trimap $t(x)$

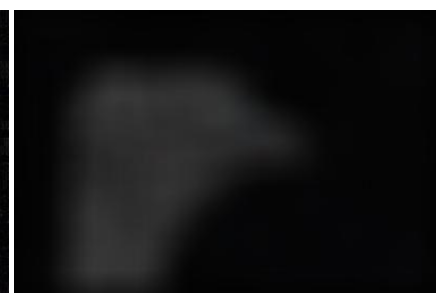

d) Local opposition $o(x)$

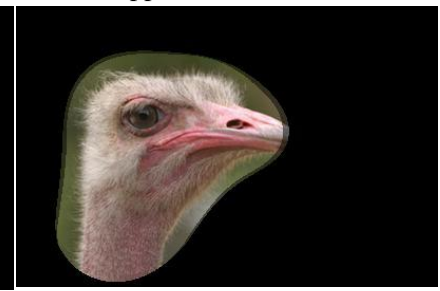

f) Extraction of salient region $e(x)$

Figure 3: Example of Inverse Bilateral Filter for Saliency Detection 


\subsection{POST-PROCESSING}

Above procedure is getting differences that induces values in small diapason. The following normalization operators are applied to the local opposition $o(x)$ and saliency $v(x)$.

$o_{n}(x)=v(o) /(\max (o)-\min (o)+\varepsilon)$

$v_{n}(v)=v(x) /(\max (v)-\min (v)+\varepsilon)$

Denote Otsu's threshold selection operator [35] by $T(u)$ :

$T(u): u(x) \rightarrow\{0,1\}$

Applying the Otsu's operator on $o_{n}(x)$ and $v_{n}(x)$ can produce a trimap $t(x)$ that is the image mask consisting of three regions of foreground, background and a blended region, where pixels are considered as a mixture of foreground and background colors [33]:

$t(x)=\left\{\begin{array}{c}1, T\left(o_{n}\right)=1 \& T\left(v_{n}\right)=1 \\ 0, T\left(o_{n}\right)=0 \& T\left(v_{n}\right)=0 \\ 0.5, \text { otherwise }\end{array}\right.$

This leads to extract salient region by the trimap $t(x)$ :

$\left\{\begin{array}{l}\Omega^{s}=\{x: t(x)=1\} \\ \Omega^{b}=\{x: t(x)=0\} \\ \Omega^{u}=\{x: 0<t(x)<1\end{array}\right\}$

$e(x)=u(x) * t(x)$

Next subsection presents algorithm for saliency detection with the inverse bilateral filter described above.

\subsection{ALGORITHM}

The algorithm IBFS is short for "Inverse Bilateral Filter for Saliency". It contains the following steps.

Start: given an input image $u(x), \sigma_{s}$ and $\sigma_{r}$.

1.Local contrast detection: define local contrast $c(x)$ by inverse bilateral filter (14).

2.Disparsity: make difference of $\mathrm{u}(\mathrm{x})$ and $\mathrm{c}(\mathrm{x})$ (16).

3.Local opposition: apply the inverse bilateral filter secondly to get $o(x)$ by (18).

4.Saliency map: get difference by (19).

5.Normalization, thresholding and creating trimap by (20), (21), (23) and (25).

Total saliency detection procedure in the IBFS algorithm includes main 4 steps, illustrated accordingly by 4 blocks in Figure 2. The inverse BF filter is applied in the first and third bloc, producing local contrast $c(x)$ and local opposition $o(x)$.

\begin{tabular}{|l|c|c|}
\hline Method & MSE & SAD \\
\hline A. SFIM [39] & 0.20 & 0.30 \\
\hline B. GBVS [40][41] & 0.21 & 0.34 \\
\hline C. RARE [42] & 0.21 & 0.33 \\
\hline D. Self-Resemblance [43] & 0.26 & 0.37 \\
\hline E. IBFS & 0.13 & 0.28 \\
\hline
\end{tabular}

Figure 4: Statistics on saliency detection for color images from [37] by methods SFIM, GBVS, RARE, Self-Resemblance and

IBFS. Error estimation metrics are Mean Squared Error (MSE) and Sum of Absolute Difference (SAD)
The second and fourth step take Euclidean distances, inducing disparity $d(x)$ and saliency $s(x)$. An input $u(x)$ from [36] in Fig.3a has its inverse BF $c(x)$ in Fig.3b. Fig.3c demonstrates the Euclidean distance $d(x)$ between $u(x)$ and $c(x)$. Fig. $3 \mathrm{~d}$ is output $o(x)$ of the third step. Saliency map $v(x)$ is in Fig.3e. Fig.3f presents normalized map $v_{n}(x)$. Finally, trimap $t(x)$ is shown in Fig.3g, followed by extraction $e(x)$ in Fig.3h.

\section{EXPERIMENTS}

To evaluate the method, a set of images in different domains from the MSRA Salient Object Database [15] is selected. Left column in Fig.5 displays original input image, the third column outlines contrast resulted by the inverse bilateral filter on the input image. Images in the fourth column are saliency maps. The second column shows extracted salient region. The column demonstrates how the IBFS works carefully with color images. It extracts intentional region including salient objects with their context.

As our algorithm produces salient region in form of trimap, Alpha Matting Evaluation database [36] with available user input trimaps is chosen to test the algorithm. Images in the first column in Fig.6 are selected from the database. The second column shows one of three trimaps input by user. Contrast map is outlined in the third column.

Salient regions are displayed in the fourth column with two error estimation metrics: Mean Squared Error (MSE) and Sum of Absolute Difference (SAD) [38]. The good reliability for average MSE covered [0.05-0.12] and SAD [0.09-0.23] in the current study compares favorably with those of previous studies for saliency matching algorithms.

Fig.7 outlines results on the same set of images from [36] by methods: Saliency for Image Manipulation (SFIM), GraphBased Visual Saliency (GBVS), the RARE model, Saliency by Self-Resemblance and the Inverse Bilateral Filter Saliency Algorithm Saliency for Image Manipulation [39] combines previously suggested patch distinctness with an object probability map. The map infers the most probable locations of the subjects of the photograph according to highly distinct salient cues.

Graph-Based Visual Saliency approach [40][41] combines multi-scale image features into a topographical saliency map. Then a dynamical neural network selects attended locations in order of decreasing saliency. The RARE model [42] uses a sequential bottom-up features extraction where first low-level features as luminance and chrominance are computed and from those results medium-level features as image orientations are extracted.

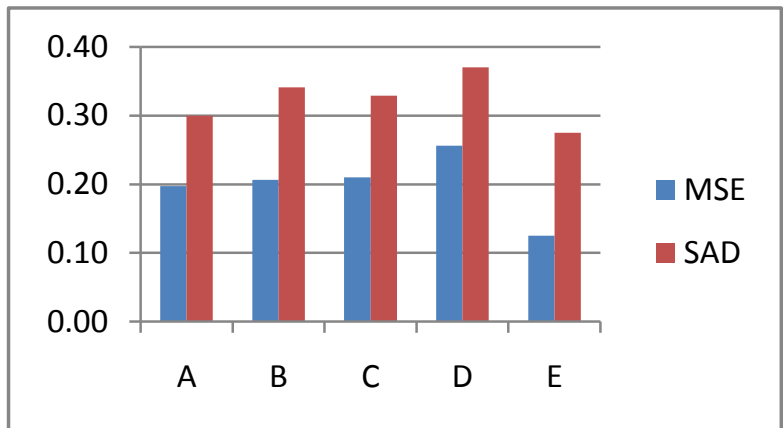


Saliency by Self-Resemblance method [43] computes socalled local regression kernels, which measure the likeness of a pixel to its surroundings. Visual saliency is then computed using self-resemblance measure resulting saliency map where each pixel indicates the statistical likelihood of saliency of a feature matrix.

The MSRA Salient Object Database [36] is used for comparative study on these methods and the IBFS. Statistics on experimental results in Fig. 5 show that IBFS keeps the best (lowest) scores on MSE and SAD for the database.

\section{DISCUSSION}

The results of the current study for the saliency estimation provide confidence that when using bilateral filter by its inverse version, contrast level can be the resource for creating saliency map. The bilateral filter has its spatial and intensity filter scales $\left(\sigma_{s}, \sigma_{r}\right)$ that manage the filter's performance.

The scales value are 1 and 0.1 in our experiments and they can be customized for each input data set. Size of filter's frame is 40 for image size $600 * 800$ in our test. As big as the size, as smooth as result but getting large time cost. Noise in input image does not affect the IBFS as the filter is self-noise remover.

\section{FUTURE WORK}

The choice of parameters for the inverse bilateral filter is still a question for further study for performance improvement.

Both stages of our algorithm finding local contrast (16) and opposition (18) rely on the filter. This allows us to have an unified concept and re-use the same filter parameters. However small or large scales may result over- or undersegmentation in estimating salient region. Current algorithm products salient region covering objects and context. Further research may focus on extraction of salient object only.

\section{CONCLUSION}

In this paper a new inverse version of bilateral filter is described and applied to generate saliency map. The inverse bilateral filter provides accurate and reliable for analyzing local contrast while remove noise and keep well original image structure. Although there is necessary to customize the filter's parameters, the inverse bilateral filter can be applicable for other applications.

\section{ACKNOWLEDGMENTS}

Author thanks Jian Sun et al for MSRA Salient Object Database [36], their images from this are used in figure 1, 3, 4 and 7. We wish to thank Christoph Rhemann et al for the Alpha Matting Evaluation Website [37], which images are exercised in figure 6 in this article.

Author would also like to thank the internal reviewers at EPU and the anonymous referees for their careful review and constructive comments.

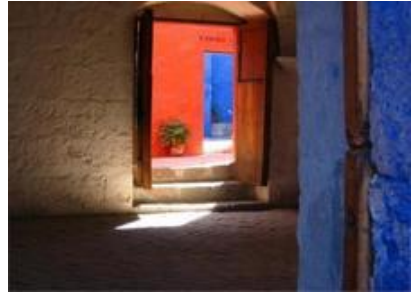

a) 192_192039.jpg

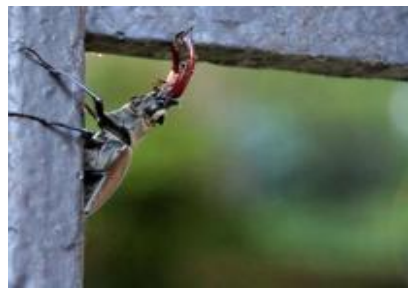

e) 192_192035.jpg

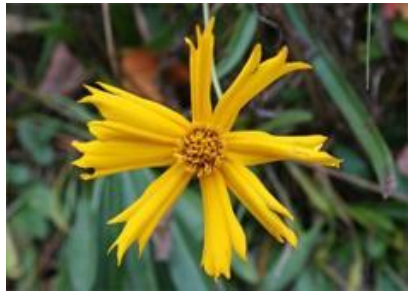

i) 192_192101.jpg

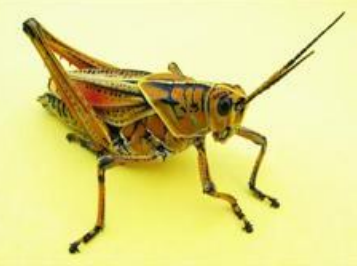

m) 192_192193.jpg

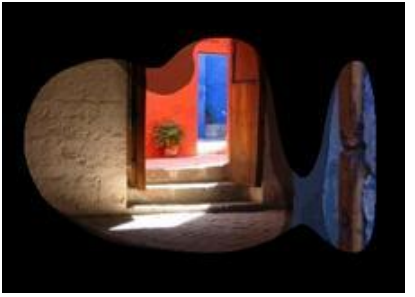

b) Extracted region

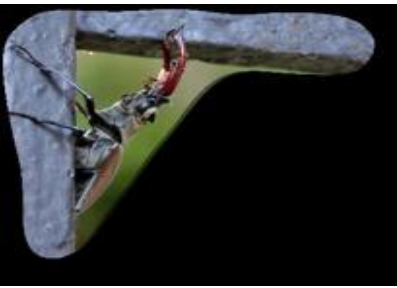

f) Extracted region

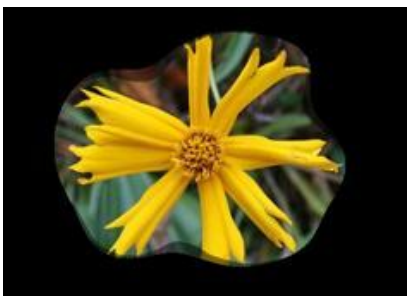

j)Extracted region

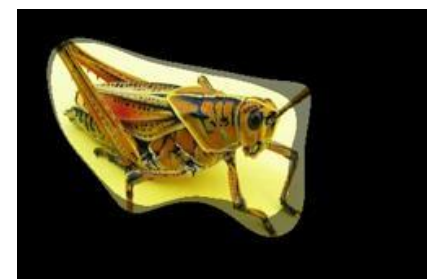

n) Extracted region

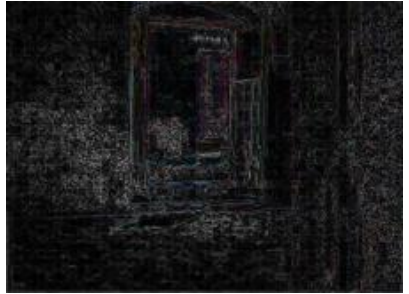

c) Contrast by inverse BF

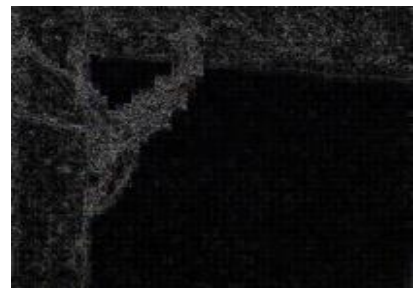

g) Contrast by inverse BF

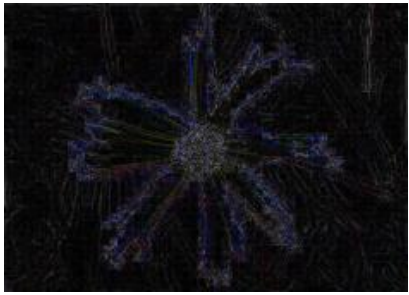

k) Contrast by inverse BF

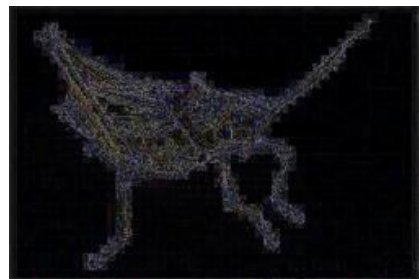

o) Contrast by inverse $\mathrm{BF}$

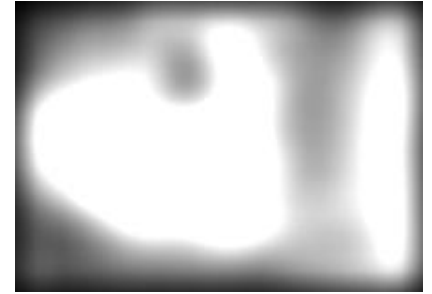

d) Saliency map

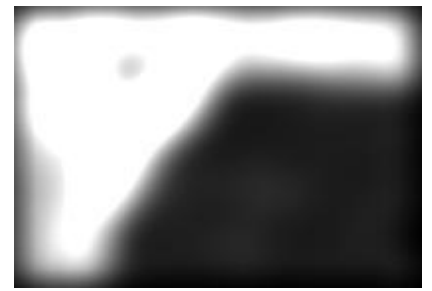

h) Saliency map

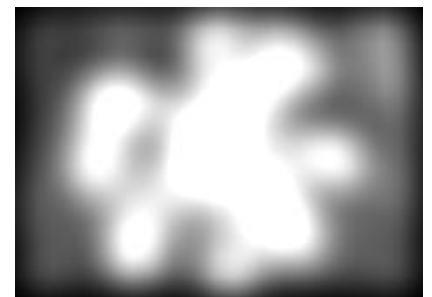

1) Saliency map

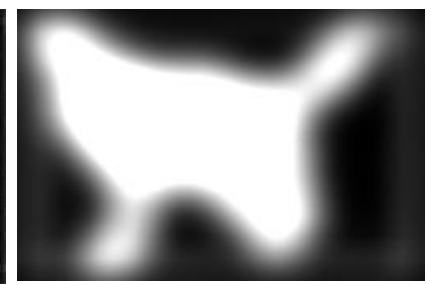

p) Saliency map 


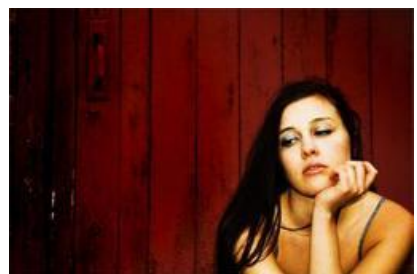

\section{q) 192_192297.jpg}

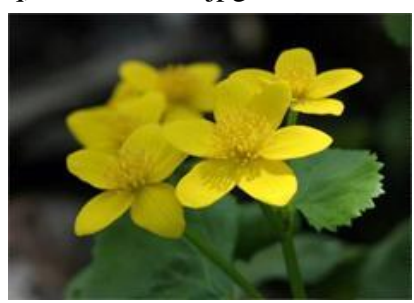

u) 192_192120.jpg

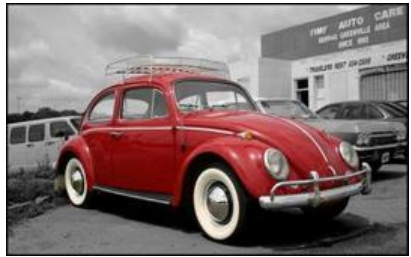

y) 195_195040.jpg

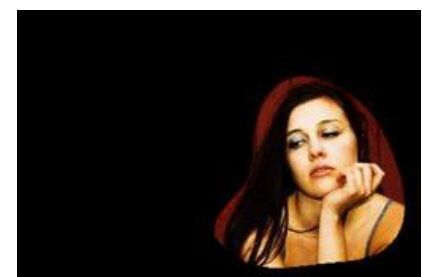

r) Extracted region

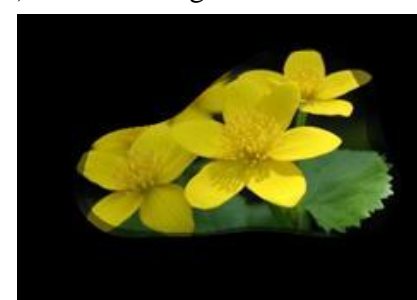

v) Extracted region

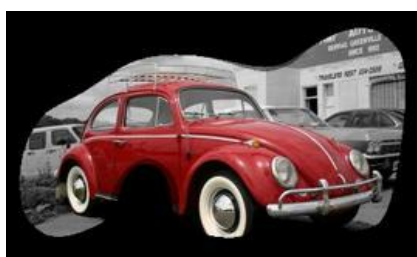

z) Extracted region

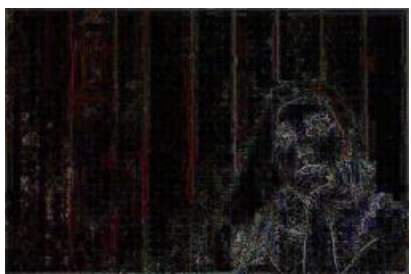

s) Contrast by inverse BF

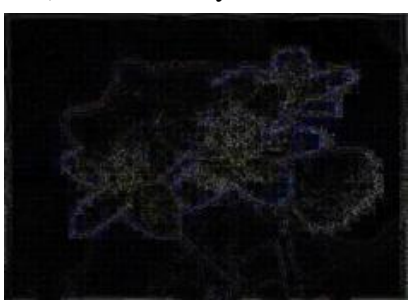

w) Contrast by inverse $\mathrm{BF}$

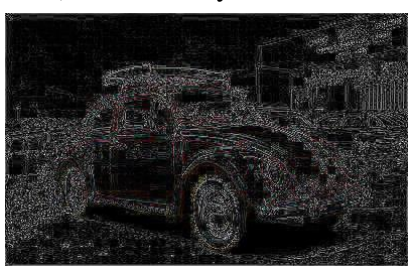

aa) Contrast by inverse BF

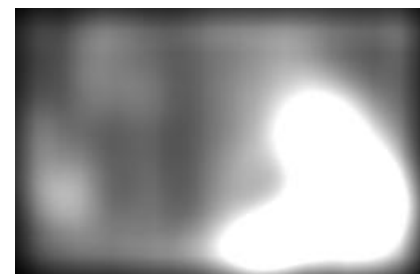

t) Saliency map

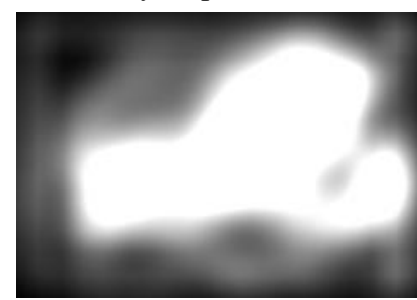

x) Saliency map

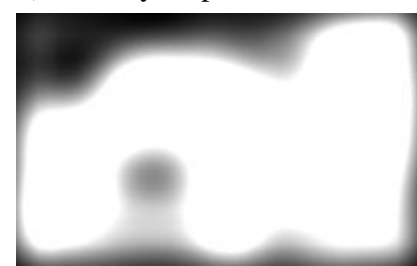

bb) Saliency map

Figure 5: Examples of saliency detection by the inverse bilateral filter for color images from the MSRA Salient Object Database [36]

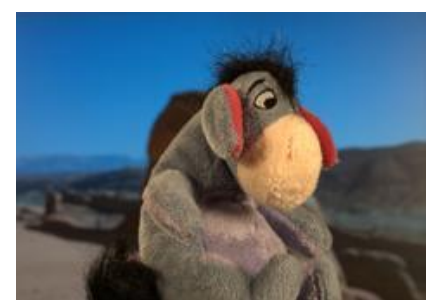

a) donkey.png

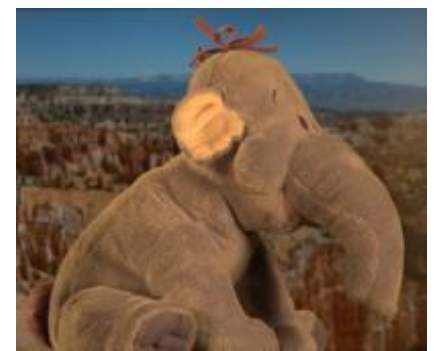

e) Elephant.png

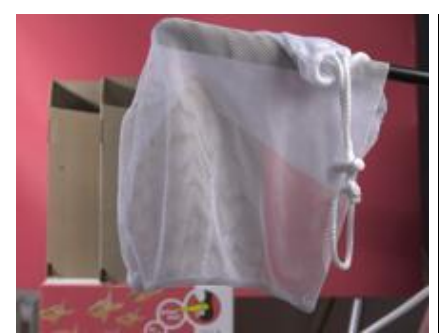

i) Net.png

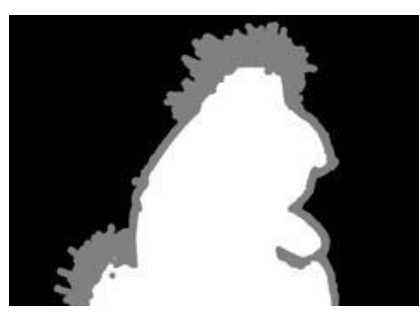

b) User input (trimap)

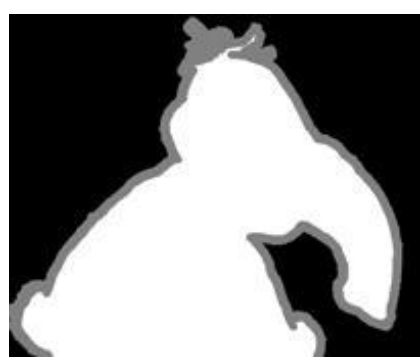

f) User input (trimap)

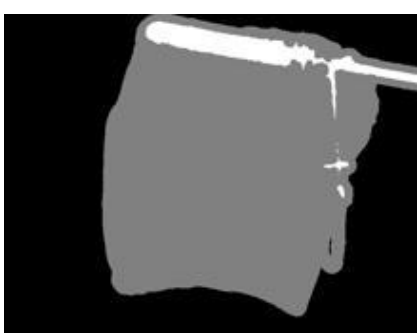

j) User input (trimap)

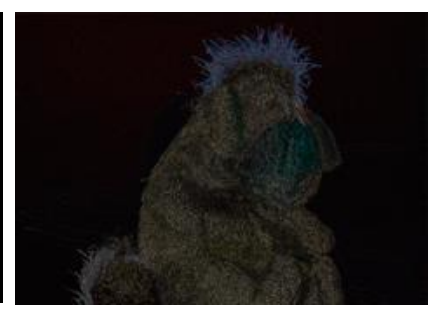

c) Contrast by IBFS

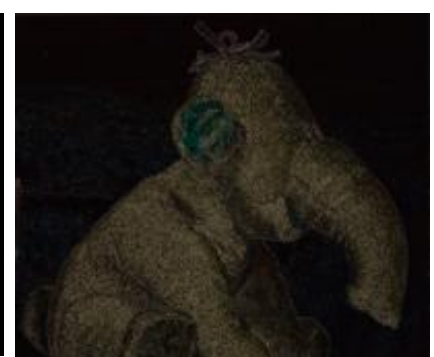

g) Contrast by IBFS

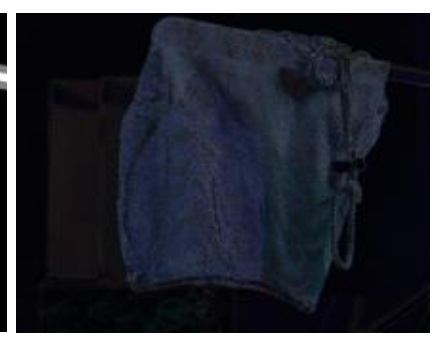

k) Contrast by IBFS

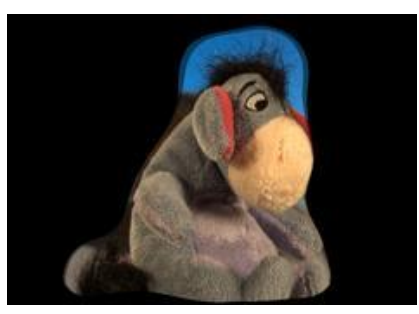

d) $\mathrm{MSE}=0.05, \mathrm{SAD}=0.09$

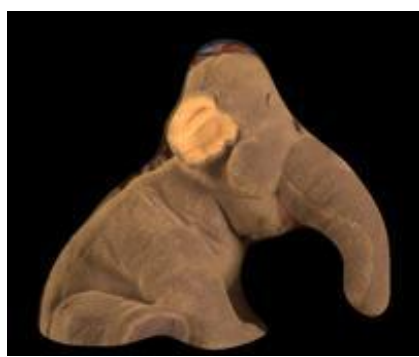

h) $\mathrm{MSE}=0.08, \mathrm{SAD}=0.12$

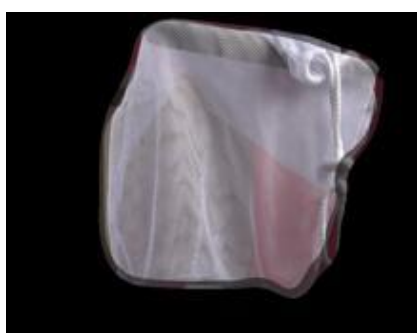

1) $\mathrm{MSE}=0.11, \mathrm{SAD}=0.23$ 


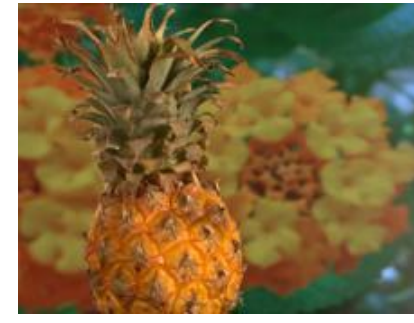

m) Pineapple.png

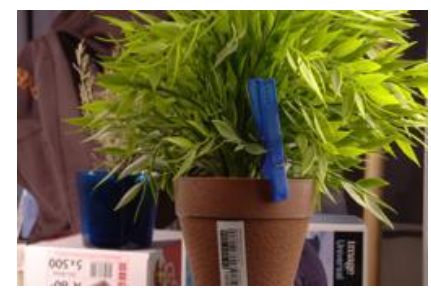

q) Plant.png

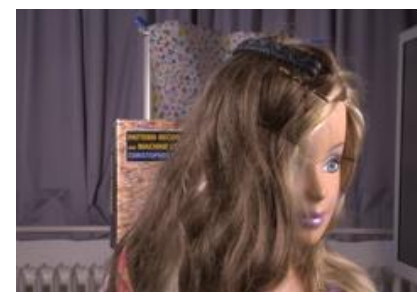

u) Doll.png

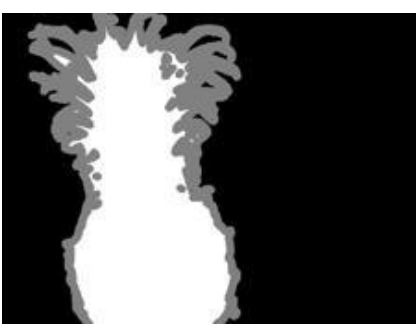

n) User input (trimap)

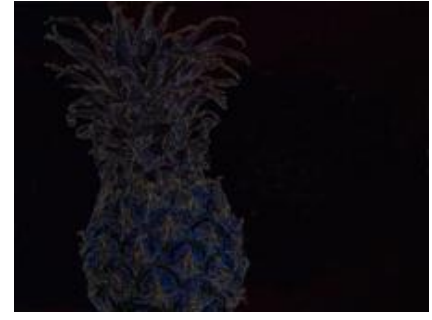

o) Contrast by IBFS

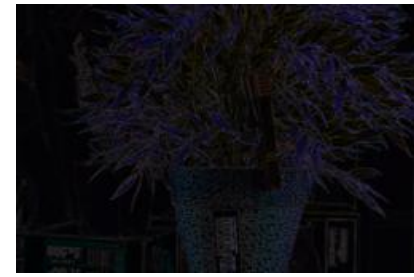

s) Contrast by IBFS

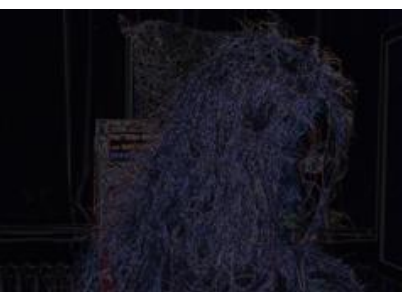

w) Contrast by IBFS

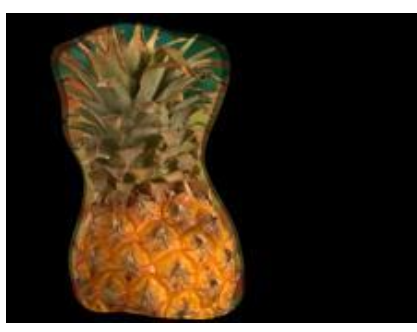

p) $\mathrm{MSE}=0.05, \mathrm{SAD}=0.09$

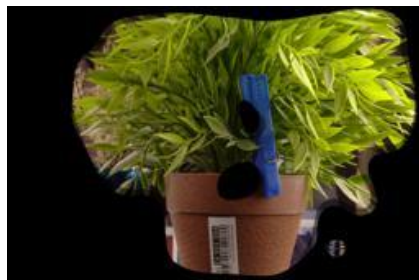

t) $\mathrm{MSE}=0.12, \mathrm{SAD}=0.20$

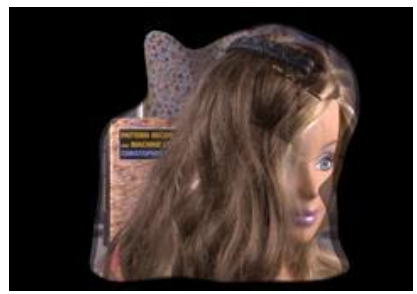

x) $\mathrm{MSE}=0.12, \mathrm{SAD}=0.17$

Figure 6: Examples of saliency detection by the inverse bilateral filter for color images from Alpha Matting Evaluation database [37]
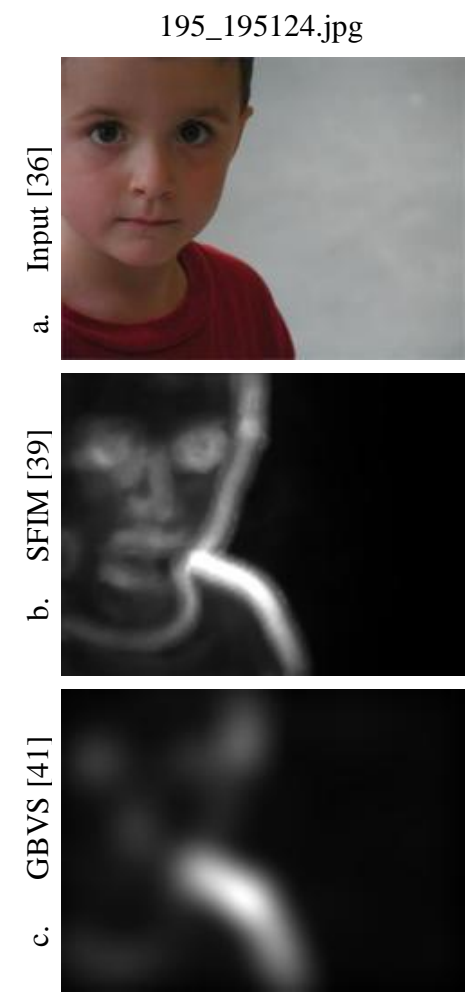

195_195371.jpg

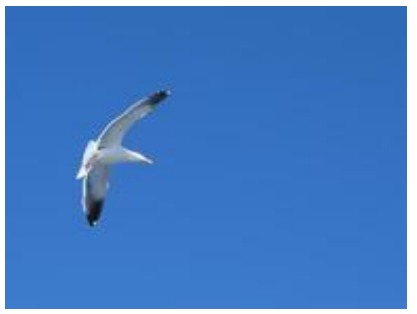

195_195501.jpg
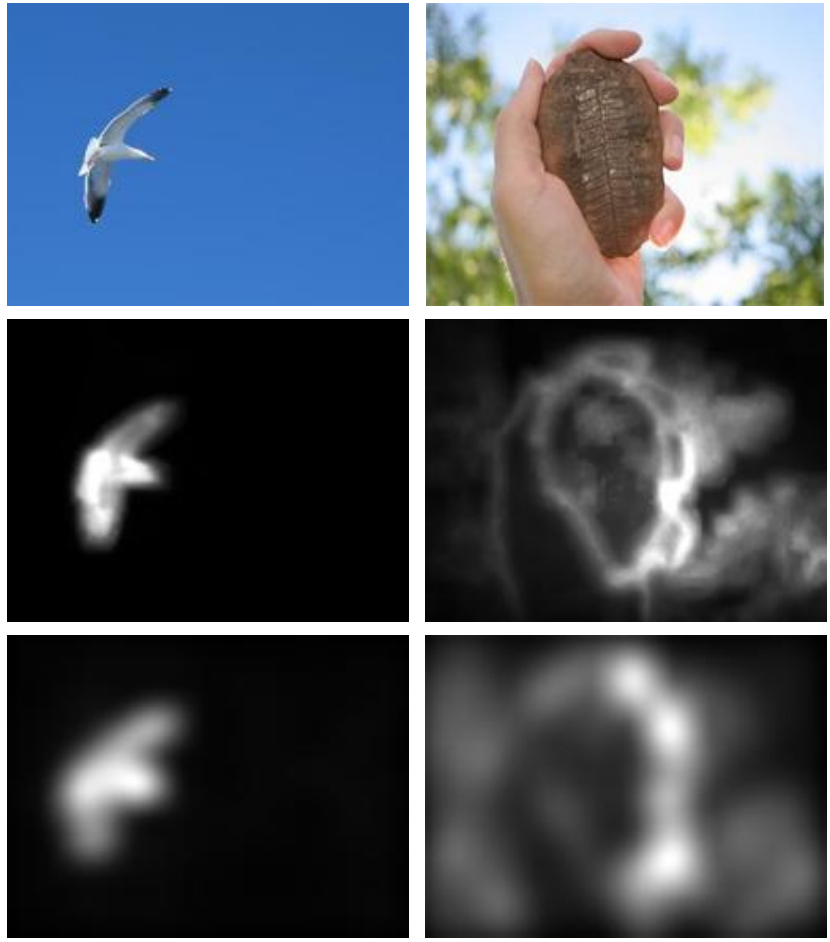
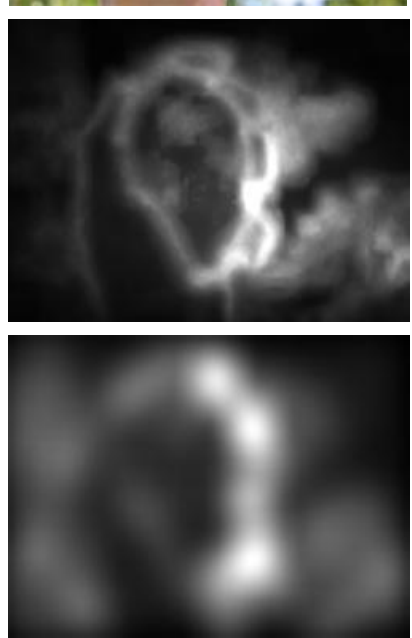

195_195008.jpg
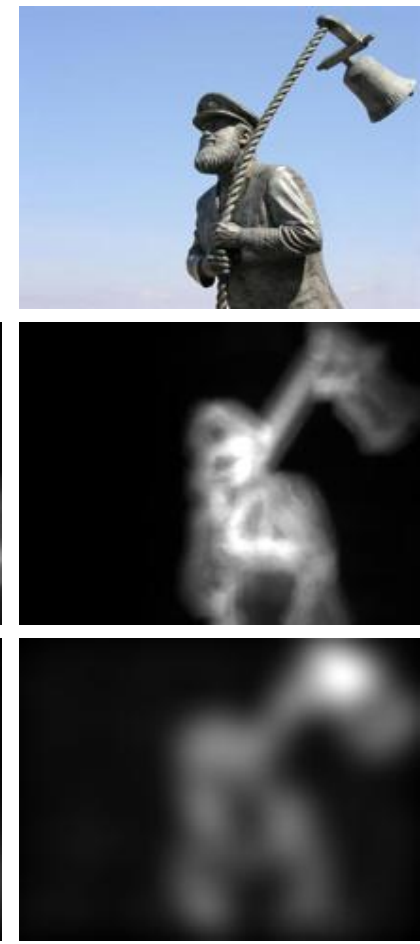

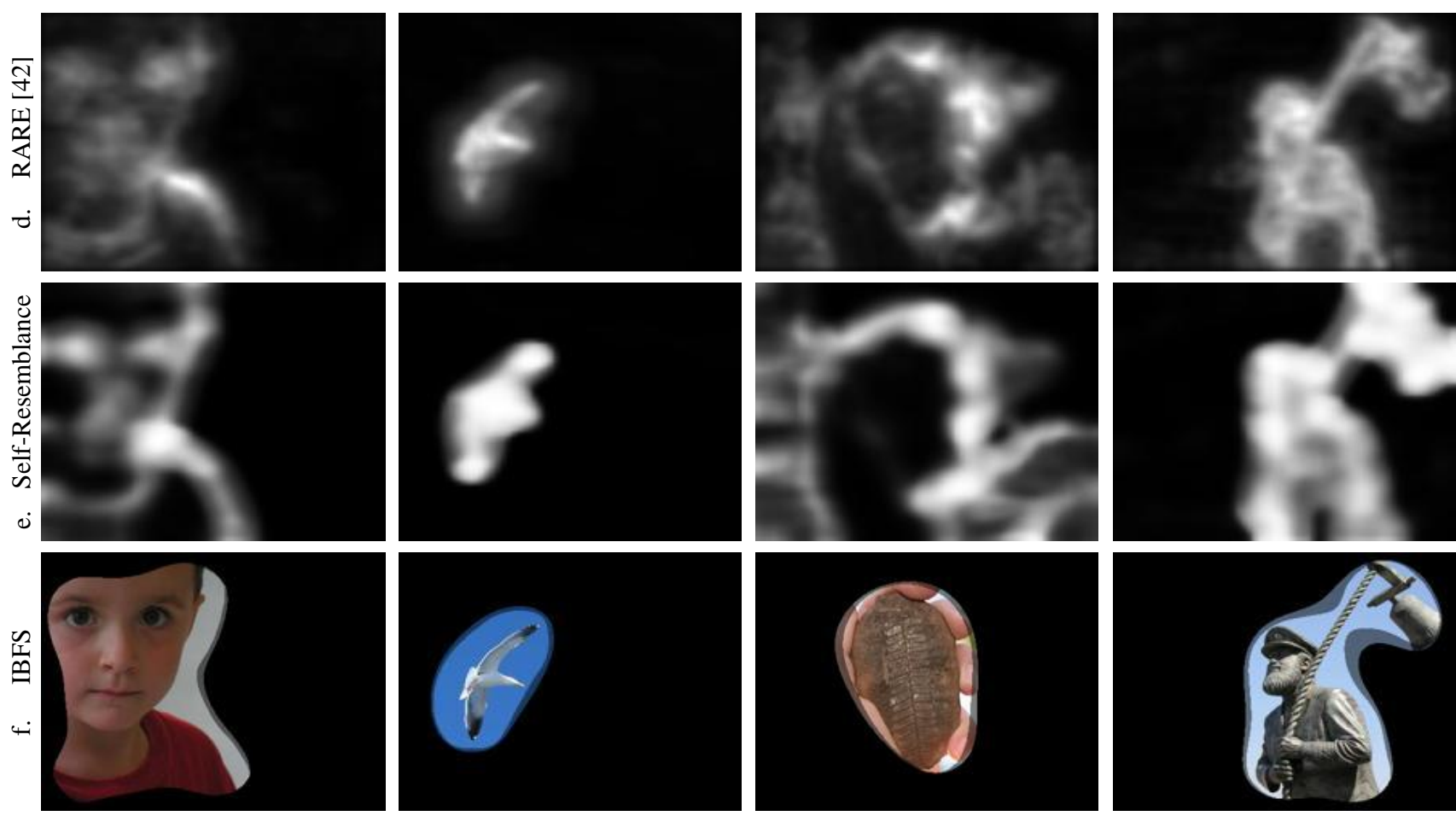

Figure 7: Examples of saliency for some images from MSRA Salient Object Database by SFIM, GBVS, RARE, Self-Resemblance and IBFS

\section{REFERENCES}

[1] Lempitsky, V., Kohli, P., Rother, C., Sharp, T.: Image segmentation with a bounding box prior. In: ICCV (2009).

[2] Marchesotti, L., Cifarelli, C., Csurka, G.: A framework for visual saliency detection with applications to image thumbnailing. In: ICCV (2009).

[3] D. Walther et al., Selective visual attention enables learning and recognition of multiple objects in cluttered scenes, Computer Vision and Image Understanding, vol. 100, nos. 1/2, pp. 41-63, 2005.

[4] S. Avidan and A. Shamir, Seam carving for contentaware image resizing, ACM Trans. Graphics, vol. 26, no. 3, pp. 1-9, 2007.

[5] Y.-F. Ma and H.-J. Zhang, Contrast-based image attention analysis by using fuzzy growing, Proc. 11th ACM Int'1 Conf. Multimedia, pp. 374-381, 2003.

[6] C. Christopoulos, A. Skodras, and T. Ebrahimi, The JPEG2000 still image coding system: an overview, IEEE Trans. Consumer Electronics, vol. 46, no. 4, pp. 11031127, Nov. 2000.

[7] C. Guo and L. Zhang, A novel multiresolution spatiotemporal saliency detection model and its applications in image and video compression, IEEE Trans. Image Processing, vol. 19, no. 1, 2010.

[8] S. Marat, M. Guironnet, and D. Pellerin, Video summarization using a visual attentional model, Proc. 15th European Signal Proc Conf., pp. 1784-1788, 2007.

[9] Michael Rubinstein, Ariel Shamir, and Shai Avidan. 2009. Multi-operator media retargeting. ACM Trans. Graph. 28, 3, Article 23 (July 2009), 11 pages.

[10] Wolf, L. Guttmann, M. ; Cohen-Or, D., Nonhomogeneous content-driven video-retargeting, Computer Vision, 2007. ICCV.
[11] Ali Borji, Dicky N. Sihite, and Laurent Itti, Salient Object detection: a benchmark, ECCV 2012.

[12] C.Tomasi, R. Manduchi. Bilateral filtering for gray and color images. Procedings of IEEE international Conf. on Computer Vision, Bombay, India, 1998: 839-846.

[13] S. Paris, P. Kornprobst, J. Tumblin and F. Durand: Bilateral filtering: theory and applications, Computer Graphics and Vision Vol 4, No.1 (2008) 1- 73.

[14] S. Paris and F. Durand, a fast approximation of the bilateral filter using a signal processing approach, Computer Science and Artificial Intelligence LaboratoryMIT, 2006.

[15] Tie Liu, Jian Sun, Nan-Ning Zheng, Xiaoou Tang and Heung-Yeung Shum. Learning to detect a salient object. In Proc. IEEE Cont. on Comp Vision and pattern Recognition (CVPR), Minneapolis, Minnesota, 2007.

[16] Treisman, A., Gelade, G., 1980. A feature-integration theory of attention. Cognitive Psychology, 12(1), pp. 97136.

[17] Laurent Itti, Christof Koch, Feature combination strategies for saliency-based visual attention systems, Journal of Electronic Imaging 10(1), 161-169, 2001 SPIE and IS\&T.

[18] S. Treue and J. C. M. Trujillo, Feature-based attention influences motion processing gain in macaque visual cortex, Nature (London) 399, 575-579, 1999.

[19] Wolfe, J. M. (1994). Guided Search 2.0: A revised model of visual search. Psych. Bull. \& Rev., 1, 202-238.

[20] Tsotsos, J.K., Culhane, S., Wai, W., Lai, Y., Davis, N., Nuflo, F., Modeling visual attention via selective tuning, Artificial Intelligence 78(1-2), p 507 - 547, 1995.

[21] Scholl B. J. (2001). Objects and attention: The state of the art. Cognition, 80 (1-2), 1-16. 
[22] Grossberg, S., Raizada, R.D., 2000. Contrast-sensitive perceptual grouping and object-based attention in the laminar circuits of primary visual cortex. Vision Res. 40, $1413-1432$.

[23] T Huang, S Grossberg, Cortical dynamics of contextually cued attentive visual learning and search: Spatial and object evidence accumulation, Technical Report CAS/CNS-TR-09-010, 2010.

[24] F Orabona1, G Metta1, G Sandini. A Proto-object Based Visual Attention Model.

[25] Jian Li and Martin D. Levine, Image and video region saliency based on space and motion, CIM25, Symposium On Brain, Body And Machine, 10-12, 2010.

[26] M Dziemianko, A Clarke, F Keller, Object-based saliency as a predictor of attention in visual tasks, Proc of the 35th An Conf of the Cognitive Science Society, 2237-2242. Berlin, 2013.

[27] D Tjondronegoro, Adaptive bilateral filtering using saliency map for de-blocking low bit rate videos, IEEE (ICME) 2014.

[28] Y Miao, R Han, H Shou, A Fast algorithm for contentaware saliency detection and stylized rendering, The 2nd Inter Conf on Computer Application and System Modeling (2012).

[29] Lu SP, Zhang SH. Saliency-based fidelity adaptation preprocessing for video coding. Journal Of Comp Sc and Tech, 26(1): 195\{202\} 2011.

[30] H. Winnemoller, S. C. Olsen, and B. Gooch, Real-time video abstraction, ACM Transactions on Graphics, vol. 25, no. 3, pp. 1221-1226, Proceedings of the ACM SIGGRAPH conference, 2006.

[31] Gokhan Yildirim, Sabine Susstrunk, FASA: fast, accurate, and size-aware salient object detection, Proceedings of the 12th Asian Conference on Computer Vision, 2014.

[32] J. Leroy, N. Riche, M. Mancas, B. Gosselin, T. Dutoit, 2014, SuperRare: an object-oriented saliency algorithm based on superpixels rarity, IEEE Inter Conf on Robotics and Automation (ICRA 2014).

[33] Olivier Juan, Renaud Keriven, Trimap segmentation for fast and user-friendly alpha matting, Lecture Notes in Computer Science Volume 3752, 2005, pp 186-197.

[34] M. Lindenbaum, M. Fischer, and A. M. Bruckstein, On Gabor's contribution to image enhancement, Pattern Recognition, 27 (1994), pp. 1-8.

[35] Otsu, N., A threshold selection method from gray-level histograms, IEEE Transactions on Systems, Man, and Cybernetics, Vol. 9, No. 1, 1979, pp. 62-66.

[36] Tie Liu, Jian Sun, Nan-Ning Zheng, Xiaoou Tang and Heung-Yeung Shum. Learning to detect a salient object. In Proc. IEEE Cont. on Comp Vision and pattern Recognition (CVPR), Minneapolis, Minnesota, 2007.

[37] Christoph Rhemann, Carsten Rother, Jue Wang, Margrit Gelautz, Pushmeet Kohli, Pamela Rott. A perceptually motivated online benchmark for image matting. CVPR 2009: 1826-1833.

[38] E. G. Richardson, Iain. H.264 and MPEG-4 Video compression: video coding for next-generation multimedia. Chichester: John Wiley \& Sons Ltd. 2003.

[39] R. Margolin, L. Zelnik-Manor, and A. Tal, Saliency for image manipulation, The Visual Computer, 2012.

[40] L. Itti, C. Koch, and E. Niebur. A model of saliencybased visual atten tion for rapid scene analysis. PAMI, 1998.

[41] Xiaodi Hou, Jonathan Harel, Christof Koch: Image signature: highlighting sparse salient regions. IEEE Trans. Pattern Anal. Mach. Intell. 34(1): 194-201 (2012)

[42] N. Riche, M. Mancas, B. Gosselin, T. Dutoit, 2012, RARE: A new bottom-up saliency model, Proc of the IEEE Inter Conf on Image Processing, USA, 2012.

[43] Hae Jong Seo and Peyman Milanfar, Nonparametric bottom-up saliency detection by self-resemblance, Journal of vision 9 (12), 15, 2009. 